\title{
Coetáneos y actitud ante el consumo de drogas en menores
}

\author{
María Lidia Platas-Ferreiro \\ Departamento de Didáctica y Organización escolar, Universidad de Santiago de Compostela, España
}

\begin{abstract}
Resumen
Las amistades y el grupo de coetáneos juegan un papel importante en la información que reciben los menores sobre las drogas y las adicciones, quienes a partir de ahí van forjando una actitud, positiva o negativa, ante su consumo. Los jóvenes que refieren tener un consumo habitual tienen una actitud más optimista frente al uso de drogas. La percepción de dicha aprobación del uso de drogas constituye un factor de riesgo que incrementa la vulnerabilidad de los menores a conductas y consumos futuros insalubres.
\end{abstract}

Palabras clave: [consumo, coetáneos, actitud, drogas, menores.]

Conocer las actitudes de los menores ante el consumo de drogas propio y ajeno y determinar la influencia que el círculo de amigos/as tiene sobre el inicio y/o mantenimiento del consumo se convierte en una tarea primordial para prevenir el consumo desde el ámbito escolar.

\section{Factores de riesgo}

El uso y abuso de las drogas, tanto legales como ilegales, constituye un problema grave debido a las alteraciones físicas, psicológicas y sociales que produce en las personas. Se necesita una acción preventiva eficaz en cuanto a la prevención del inicio de consumo de drogas por muchas razones, entre las que destacan según Schioler (1991) el gran descenso en la edad de inicio de consumo, la extensión del consumo a todas cas capas sociales, el tipo de sustancias y la frecuencia del consumo.

La actitud hacia el consumo de drogas es medida por ítems relacionados con una disposición más favorable al consumo. La OMS da prioridad a la prevención de consumo sobre los grupos más expuestos a las drogodependencias: menores y adolescentes. Nos encontramos en un momento de riesgo elevado para niños, adolescentes y jóvenes en el que la prevención se convierte en una acción prioritaria (Vega, 1983).

Fortalecer las actitudes contrarias al consumo de alcohol u otras drogas, o el cambio de actitudes neutrales a contrarias resulta más fácil que revertir las actitudes positivas una vez que se ha empezado a consumir. La influencia de los amigos tiene una especial fuerza en la iniciación en el consumo de tabaco y marihuana. Los cambios de actitudes siguen siendo la meta de muchos programas escolares de prevención y campañas de los medios de comunicación (Platas, 2009).

\section{El consumo en menores}

Platas (2012) señala en el II Plan Municipal de Drogodependencias, que una de las líneas estratégicas de actuación del Plan de Trastornos Adictivos de Galicia 2011-2016 es la reducción de la prevalencia de los consumos de bebidas alcohólicas, tabaco y otras drogas ilícitas en menores. La realidad es que casi el $70 \%$ de los alumnos/as de Primaria y un $85 \%$ del alumnado de la Eso y Bac tienen claro que el alcohol y el tabaco causan problemas de salud. Las edades a las que se ha consumido por primera vez, y el tipo de sustancias consumidas se reflejan en la siguiente tabla. (Véase Tabla 1)

Tabla 1.

Sustancias y edades del primer consumo

\begin{tabular}{lcccc}
\hline $\begin{array}{l}\text { Edad } \\
\text { (años) }\end{array}$ & $\begin{array}{c}\text { Alcohol } \\
(\%)\end{array}$ & $\begin{array}{c}\text { Porros } \\
(\%)\end{array}$ & $\begin{array}{c}\text { Cocaína } \\
(\%)\end{array}$ & $\begin{array}{c}\text { Tabaco } \\
(\%)\end{array}$ \\
\hline 11 & 5,75 & 0,50 & 0,19 & 2,96 \\
$12-13$ & 14,35 & 2,45 & 0,31 & 9,13 \\
$14-15$ & 24,80 & 7,68 & 0,76 & 11,83 \\
$16-17$ & 6,80 & 4,47 & 0,76 & 5,65 \\
\hline
\end{tabular}

Las cifras son clarificadoras de los retos que debemos plantearnos: el alcohol es la droga legal más consumida y las edades de inicio son realmente preocupantes, sobre todo teniendo en cuenta que el proceso de desarrollo de los menores está aún inacabado. Por tanto, según Platas (2010) reducir la demanda de consumo de alcohol en menores, sigue siendo uno de los objetivos prioritarios de la Delegación del Gobierno para el Plan Nacional sobre Drogas en su Estrategia Nacional 2009-2016 y del Plan Municipal de Lugo sobre Drogodependencias durante el período 2012-2016.

\section{Objetivos}

Describir las relaciones entre el consumo de drogas en el ámbito de ocio y el de los menores analizando cómo las amistades influyen y determinan el consumo y su actitud ante las drogas.

\section{Método}

Estudiantes de Primaria de la capital lucense que han respondido a una encuesta que pretendía recoger información sobre los hábitos de consumo, la actitud y el conocimientos que estos menores tienen hacia las drogas en general y cómo la actitud, el conocimiento y el consumo de sus amigos pueden influirles de una forma más o menos determinante. 


\section{Participantes}

822 estudiantes de la capital lucense (398 niñas y 414 niños) que cursan $5^{\circ}(373)$ y/o $6^{\circ}(449)$ de primaria, todos entre 10 y 12 años de edad.

24 colegios que ofertan Educación primaria en la capital lucense; esta cantidad se corresponde con el 100\% de centros educativos. (Véase Tabla 2)

Tabla 2.

Colegios participantes de la capital lucense

\begin{tabular}{lcc}
\hline Colegios de la capital lucense & CEIP & CPR \\
\hline Albeiros & $\mathrm{X}$ & \\
Virxe da Soidade & $\mathrm{X}$ & \\
Mercedes & $\mathrm{X}$ & \\
Anexa & $\mathrm{X}$ & \\
A Ponte & $\mathrm{X}$ & \\
Quiroga Ballesteros & $\mathrm{X}$ & \\
Sagrado Corazón & $\mathrm{X}$ & \\
\hline Rosalía de Castro & $\mathrm{X}$ & \\
Casás & $\mathrm{X}$ & \\
\hline Luis Pimentel & $\mathrm{X}$ & \\
Paradai & $\mathrm{X}$ & \\
\hline Menéndez Pelayo & $\mathrm{X}$ & \\
Illa Verde & $\mathrm{X}$ & \\
\hline Cervantes & & $\mathrm{X}$ \\
Salesianos & & $\mathrm{X}$ \\
Divino Maestro & & $\mathrm{X}$ \\
\hline Fingoy & & $\mathrm{X}$ \\
Galén & & $\mathrm{X}$ \\
\hline A Inmaculada & $\mathrm{X}$ \\
A Milagrosa & & $\mathrm{X}$ \\
María Auxiliadora & & $\mathrm{X}$ \\
\hline Franciscanos & & $\mathrm{X}$ \\
San José & & \\
\hline Seminario Diocesano & & \\
\hline
\end{tabular}

\section{Materiales}

Se ha elaborado una encuesta sobre hábitos, actitudes $\mathrm{y}$ conocimientos acerca de las drogas, y se han analizado un total de 27.126 ítems

\section{Resultados}

Los resultados encontrados confirman que 185 jóvenes han probado el alcohol (un 22,51\% del total de los encuestados). El 65\% de los menores pasan a mayor parte de su tiempo libre con amigos y amigas de la misma edad y el $26 \%$ con amistades de todas las edades.

Por otra parte, del total de encuestados/as sólo 4 de cada 100 han probado el tabaco $(\mathrm{N}=33)$ y de ellos un $57 \%$ lo han conseguido en sus propias casas o en casa de sus amigos, un $25 \%$ en la calle y un $10 \%$ en un bar. Sea donde fuere, un $30 \%$ lo ha conseguido y consumido con sus amigos/as . Un 50\% de los menores que se iniciaron en el consumo de tabaco tienen amigos de su misma edad y un tercio de estos menores tienen amigos de todas las edades. Este patrón se repite con el consumo de alcohol: el $50 \%$ de los menores que cursan $5^{\circ}$ y $6^{\circ}$ de primaria y que dicen haber consumido alcohol, afirman que pasan más tiempo con amigos de su misma edad, mientras que un $25 \%$ suelen ir acompañados de amigos de todas las edades.

Un $80 \%$ de los menores del último ciclo de Primaria, tienen una imagen muy negativa del consumo de drogas, siendo que al $20 \%$ restante o le parece bien o le es indiferente.

En cuanto a si son influenciables o no por sus amistades, se encontró que casi un $94 \%$ reconoce que no se dejaría convencer por sus amigos/as para consumir drogas e incluso un $60 \%$ afirma que estaría dispuesto a cambiar de amistades si esto llegara a ocurrir.

El 100\% de los encuestados reconocen como princiapl motivo para iniciarse en el consumo, la curiosidad. Sin embargo, como motivos secundarios se encontró (sólo en varones) que otras razones han sido la influencia de los demás.

Además, un $85 \%$ estaría dispuesto a hablar con sus amigos y conocidos acerca de los peligros que comporta el consumo de drogas y en el mismo porcentaje dejarñian de ir a aquellos lugares (parques, plazas, cafeterías...) donde sepan que sus conocidos están consumiendo drogas.

\section{Conclusiones}

Los resultados muestran como estas edades son especialmente peligrosas para el inicio (y mantenimiento) del consumo de drogas: $6^{\circ}$ de primaria es uno de los cursos que conlleva más riesgos al ser el último curso de primaria y servir de punete para acceder a la Eso.

Los resultados muestran además, que la amistad tiene, en esta edad, un valor excepcional, lo que podría servir no sólo a nivel explicativo para insistir en la influencia y efectos de la presión de grupo, sino además para replantearse y rediseñar intervenciones basadas precisamente en la capacidad de los preadolescentes para prevenir el consumo entre sus iguales y en la labor diagnóstica y preventiva del profesorado y la familia.

No parece haber sido decisiva la edad de los amigos/as para influenciar el consumo de los menores. Pese a lo que se podría esperar, los menores cuando coquetean con las drogas lo hacen en compañía de otros menores de su misma edad.

La quinta parte de los menores que han admitido estar a favor del consumo de drogas o serle indiferente se convierte en una población diana para trabajar la prevención del inicio y/o mantenimiento en el consumo, ya que ellos/as podrían ser además de consumidores, futuros influenciadores en los demás.

A raíz de los resultados obtenidos, sobre todo en cuanto a la disponibilidad de los menores para hablar y convencer a otros menores de los peligros del consumo de drogas, deberíamos pensar en otras posibles estrategias de prevención, donde contemos con el protagonismo de los propios jóvenes como mediadores, sensibilizadores y comunicadores. 


\section{Referencias}

Platas, M.L. (2009): Familias, jóvenes, drogas y tiempo libre. Lugo: Concello de Lugo

Platas, M.L. et al (2010): Estado de situación de tratamiento para problemas por consumo de drogas en el ámbito local: información de 19 ciudades de Europa, América Latina y El Caribe. Coímbra, Portugal: Organización de los Estados Americanos

Platas, M.L. (2012): II Plan Municipal de Prevención de Drogodependencias de Lugo, 2012-2015. Lugo: Concello de Lugo

Platas, M.L. (2012): Jóvenes y familias ante las drogas. Lugo: Concello de Lugo

Schioler, P. (1991). Estrategias de prevención de los problemas de origen etílico en los estados miembros de la Comunidad Europea. Revista española de Drogodependencias, 16(1), 45-49.

Vega, A. (1983). Más allá de la escuela o la educación comunitaria. Revista española de Drogodependencias, 16(2), 103-113.

Navarro, J. (2000). Factores de riesgo y protección de carácter social relacionados con el consumo de drogas. Madrid: Ayuntamiento de Madrid.

\section{Agradecimientos}

A todos los centros educativos públicos, privados y concertados de la capital lucense

A todos los jóvenes lucenses que han respondido a esta encuesta

A todas las familias de dichos jóvenes por aceptar su participación en esta investigación

A los profesores/as por su sensibilidad con la prevención de consumo de drogas entre el alumnado del último ciclo de primaria. 